\title{
Strain-related phenomena in GaN thin films
}

\author{
C. Kisielowski, ${ }^{*}$ J. Krüger, S. Ruvimov, T. Suski, J. W. Ager III, E. Jones, Z. Liliental-Weber, M. Rubin, and E. R. Weber \\ Lawrence Berkeley National Laboratory and University of California, Berkeley, California 94720 \\ M. D. Bremser and R. F. Davis \\ Department of Materials Science and Engineering, North Carolina State University, Raleigh, North Carolina 27695
}

(Received 28 June 1996)

\begin{abstract}
Photoluminescence (PL), Raman spectroscopy, and x-ray diffraction are employed to demonstrate the coexistence of a biaxial and a hydrostatic strain that can be present in $\mathrm{GaN}$ thin films. The biaxial strain originates from growth on lattice-mismatched substrates and from post-growth cooling. An additional hydrostatic strain is shown to be introduced by the presence of point defects. A consistent description of the experimental results is derived within the limits of the linear and isotropic elastic theory using a Poisson ratio $\nu=0.23 \pm 0.06$ and a bulk modulus $B=200 \pm 20 \mathrm{GPa}$. These isotropic elastic constants help to judge the validity of published anisotropic elastic constants that vary greatly. Calibration constants for strain-induced shifts of the near-bandedge PL lines with respect to the $E_{2}$ Raman mode are given for strain-free, biaxially strained, and hydrostatically contracted or expanded thin films. They allow us to extract differences between hydrostatic and biaxial stress components if present. In particular, we determine that a biaxial stress of one GPa would shift the near-band-edge PL lines by $27 \pm 2 \mathrm{meV}$ and the $E_{2}$ Raman mode by $4.2 \pm 0.3 \mathrm{~cm}^{-1}$ by use of the listed isotropic elastic constants. It is expected from the analyses that stoichiometric variations in the GaN thin films together with the design of specific buffer layers can be utilized to strain engineer the material to an extent that greatly exceeds the possibilities known from other semiconductor systems because of the largely different covalent radii of the $\mathrm{Ga}$ and the $\mathrm{N}$ atom. [S0163-1829(96)03148-7]
\end{abstract}

\section{INTRODUCTION}

In recent years gallium nitride has attracted considerable attention because of the materials potential to fabricate bright UV- and visible-light-emitting diodes, power devices, and lasers. ${ }^{1-3}$ Efforts in thin-film growth led to a substantial improvement in heteroepitaxially grown GaN films. ${ }^{4}$ Homoepitaxy became possible very recently by use of bulk-grown GaN as a substrate material. ${ }^{5,6}$ The material's ability to tolerate unusually large extended defect densities $\left(10^{8} \mathrm{~cm}^{-2}\right.$ and larger) without deterioration of physical properties such as the electroluminescence performance, allows for an unprecedented technical progress. Details of the underlying physical processes, however, are not yet well understood.

Experimentally, GaN thin films and bulk crystals are often grown under extreme conditions: e.g., bulk GaN is grown around $1500{ }^{\circ} \mathrm{C}$ under a hydrostatic nitrogen pressure of $1-2$ $\mathrm{GPa}$; molecular-beam epitaxy $(\mathrm{MBE})$ growth is performed at relative low temperatures $\left(\sim 700{ }^{\circ} \mathrm{C}\right)$ to benefit from deviations in a thermodynamic equilibrium; the most commonly used substrates for heteroepitaxy are sapphire and $\mathrm{SiC}$ that may introduce large strain into the GaN layers because of a lattice mismatch of $-13 \%$ and $+4 \%$, respectively. ${ }^{4}$ Thus, strain and deviations from a stoichiometric composition of GaN certainly must be considered in order to understand the material's properties. Theoretical calculation performed up to now ${ }^{7,8}$ do not yet account for this fact. They stress, however, that the native defect formation in $\mathrm{GaN}$ is strongly ruled by strain because of the significantly different covalent radii of the $\mathrm{Ga}$ and the $\mathrm{N}$ atoms. ${ }^{7}$ Experimentally, large variations of the lattice parameters were observed and attributed to the incorporation of impurities and of nonstoichio- metric native defects into the GaN lattice. ${ }^{9}$ Very recently, a Ga supersaturation concentration was reported in bulk grown $\mathrm{GaN}$ crystals that may lead to the formation of Ga-related defects or defect clusters such as precipitates of varying size or even $\mathrm{Ga}_{\mathrm{N}}$ antisite defects in large concentrations $(>1 \%$ locally). ${ }^{10}$ Moreover, it was realized that the thermal, biaxial strain introduced by different thermal expansion coefficients of the substrates and the GaN films introduces stresses that can lead to cracking of the brittle material, ${ }^{11}$ which hardly allows for plastic deformation. However, the presence of the thermal biaxial strain and the hydrostatic strain induced by point defects are treated up to now as if they are separate issues that do not influence each other.

It is the purpose of this paper to point out the coexistence of the hydrostatic and the biaxial strain in GaN thin films. The external biaxial strain originates from the growth on lattice mismatched substrates and from the different thermal expansion coefficients of the GaN films and the substrates. Point defects introduce an internal hydrostatic strain that can be compressive or expansive depending on the size of the involved atoms. The strain is altered by the presence of buffer layers and of extended defects. Photoluminescence (PL), Raman, and X-ray measurements are exploited to monitor strain. Results from GaN homoepitaxy are used to obtain a zero strain calibration and the lattice parameters of the most perfect $\mathrm{GaN}$ films.

\section{STRESS-STRAIN RELATIONS FOR COEXISTING HYDROSTATIC AND BIAXIAL STRAIN FIELDS}

Principally, all necessary stress-strain relations for uniaxial, biaxial, and hydrostatic compression or tension can 
TABLE I. Anisotropic and isotropic elastic constants considered in this paper. References are indicated. $B$ is the bulk modulus, $E$ is the Young modulus, $\nu$ is the Poisson ratio. The listed isotropic elastic constants were calculated in the Voigt average (Ref. 36) from the $c_{i j}$. Our best estimatuion of the experimentally determined isotropic elastic constants and of lattice parameters of GaN are listed too.

\begin{tabular}{|c|c|c|c|c|c|c|c|c|}
\hline \multicolumn{5}{|c|}{ Anisotropic } & \multicolumn{3}{|l|}{ Isotropic } & \multirow[t]{2}{*}{ Ref. } \\
\hline$c_{11}$ & $c_{12}$ & $c_{13}$ & $c_{33}$ & $c_{44}$ & $B$ & $E$ & $\nu$ & \\
\hline 296 & 130 & 158 & 267 & 24 & 190 & 148 & 0.37 & 12 \\
\hline 396 & 144 & 64 & 476 & 91 & 201 & 314 & 0.24 & 13 \\
\hline 369 & 94.2 & 66.7 & 397 & 118 & 180 & 324 & 0.20 & 14 \\
\hline 390 & 145 & 106 & 398 & 105 & 210 & 305 & 0.26 & 15 \\
\hline \multicolumn{5}{|c|}{ Experimental values } & $200 \pm 20$ & 290 & $0.23 \pm 0.06$ & $15-18$ \\
\hline \multicolumn{5}{|c|}{ Lattice constants (nm) } & \multicolumn{2}{|c|}{$a=0.31878 \pm 0.00003$} & $c=0.51850 \pm 0.00002$ & 18 \\
\hline
\end{tabular}

be obtained if the elastic constants of GaN were known within reasonable limits. However, this is not the case for GaN. Table I lists the published anisotropic elastic constants that vary greatly. For example, Detchprohm et al. ${ }^{16}$ showed that for biaxial strain the ratio $\varepsilon_{c} / \varepsilon_{a}$ is given by $2 s_{11} /\left(s_{11}+s_{12}\right)$ where $\varepsilon_{c}$ and $\varepsilon_{a}$ are the strains along the GaN $a$ and $c$ axes and the $s_{i j}$ are the elastic compliances. In terms of elastic constants the ratio reads $\varepsilon_{c} / \varepsilon_{a}=-2 c_{13} / c_{33}$. Numerically, the different elastic constants from Table I give values $\varepsilon_{c} / \varepsilon_{a}=-1.18,{ }^{12}-0.27,{ }^{13}-0.33,{ }^{14}$ and -0.53 (Ref. 15) that vary by a factor of 4 .

In contrast, the isotropic elastic constants can be determined within moderate uncertainties. We review literature data to find values for the bulk modulus $B$ and the Poisson ratio $\nu$. A bulk modulus $B=200 \pm 20 \mathrm{GPa}$ can readily be extracted from recent publications. ${ }^{17,18}$ The Poisson ratio is related to the quotient $\varepsilon_{c} / \varepsilon_{a}=\left[\left(c-c_{0}\right) / c_{0}\right] /\left[\left(a-a_{0}\right) / a_{0}\right]$. Here, $a$ and $c$ are the strained lattice constants of $\mathrm{GaN}$ and $a_{0}, c_{0}$ are reference values for stoichiometric and defect-free GaN crystals. To our knowledge, recent lattice parameter measurements of homoepitaxially grown GaN films ${ }^{18}$ provide the most reliable values $a_{0}$ and $c_{0}$. They are listed in Table I. These numbers are used to calculate $\varepsilon_{c} / \varepsilon_{a}$ from lattice parameter measurements published in Refs. 9, 16, and 17. Figure 1 summarizes the results. We find values $1.5>\varepsilon_{c} / \varepsilon_{a}>-1.5$. Also, very few data exhibit values

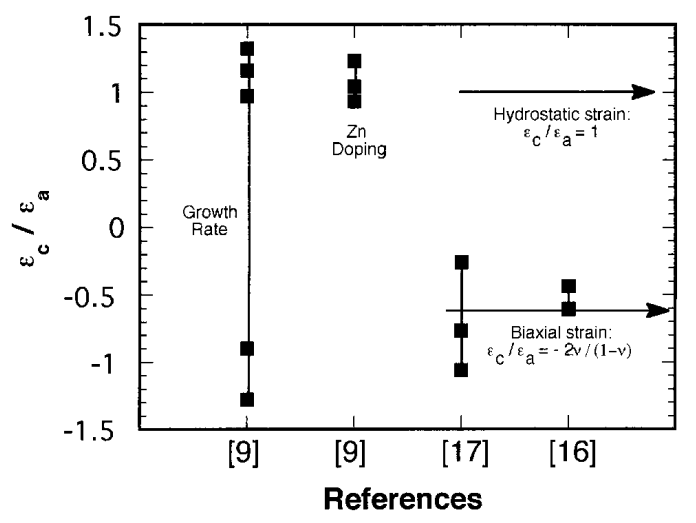

FIG. 1. Evaluation of literature data $\varepsilon_{c} / \varepsilon_{a}$. References are given. A mean value $\varepsilon_{c} / \varepsilon_{a}$ is calculated from samples that exhibit a large biaxial stress. It can be seen that the literature data exceed both limits given by a pure hydrostatic strain and a dominant biaxial strain. The values are influenced by doping and by the rate at which the GaN films are grown. $0<\varepsilon_{c} / \varepsilon_{a}<-0.5$ that one would expect if $\varepsilon_{c} / \varepsilon_{a}$ would equal the Poisson ratio. In fact, values $\varepsilon_{c} / \varepsilon_{a}$ from biaxially strained films are sometimes confused with the Poisson ratio. ${ }^{19}$ If the films were biaxially strained only, one would expect a constant value $\varepsilon_{c} / \varepsilon_{a}$. If we make the crucial assumption that in samples with a dominant biaxial strain ${ }^{16,17}$ the different hydrostatic strain components average to zero, a mean value $\varepsilon_{c} / \varepsilon_{a}=-0.6 \pm 0.2$ is calculated from Fig. 1 . This number will be used to determine the Poisson ratio. Thus, the reviewed strain ratios exceed both the limit given by a hydrostatic compression $\left(\varepsilon_{c} / \varepsilon_{a}=1\right)$ and the limit given by a dominant biaxial strain. On the other hand, the data from Ref. 9 in Fig. 1 show that the growth rate or the doping with $10^{20} \mathrm{~cm}^{-3}$ of $\mathrm{Zn}$ initiates the changes in $\varepsilon_{c} / \varepsilon_{a}$. Thus, it seems that nonstoichiometric growth or the incorporation of atoms that differ in size from the $\mathrm{Ga}$ and the $\mathrm{N}$ atom causes the deviation of $\varepsilon_{c} / \varepsilon_{a}$ from a constant mean value that we expect only if the samples were biaxially strained.

Therefore, we choose to describe the coexistence of a biaxial and a hydrostatic strain field in terms of isotropic elastic theory. In this approximation the relation between stress and strain is ${ }^{20}$

$$
\varepsilon_{i}=E^{-1}\left[\sigma_{i}-\nu\left(\sigma_{j}+\sigma_{k}\right)\right],
$$

where $i, j, k$ label the stresses and the strains in the basal plane $a, b$ and along the $c$ axis. Shear components are neglected. In case of hydrostatic pressure $\left(\sigma_{a}=\sigma_{b}=\sigma_{c}=\sigma_{p}\right)$ Eq. (1) reduces to

$$
\varepsilon_{p}=E^{-1} \sigma_{p}(1-2 \nu),
$$

and the ratio $\varepsilon_{i} / \varepsilon_{j}$ is one. For the case of a biaxial stress in the basal plane $\left(\sigma_{a}=\sigma_{b}, \sigma_{c}=0\right)$ the relations are

$$
\begin{gathered}
\varepsilon_{a}=E^{-1} \sigma_{a}(1-\nu), \\
\varepsilon_{c}=-E^{-1} \sigma_{a} 2 \nu, \\
\varepsilon_{c} / \varepsilon_{a}=-2 \nu /(1-\nu) .
\end{gathered}
$$

It is clear from Eq. (3c) that for a biaxial stress the measured lattice constants $c$ and $a$ are related to the Poisson ratio by $\left[\left(c-c_{0}\right) / c_{0}\right] /\left[\left(a-a_{0}\right) / a_{0}\right]=-2 \nu /(1-\nu)$. We use the value $\varepsilon_{c} / \varepsilon_{a}=0.6 \pm 0.2$ from Fig. 1 to determine $\nu=0.23 \pm 0.06$.

Strain induced by substitutional point defects can be introduced into the model considering Vegard's law that can be expressed by the equation 


$$
\varepsilon=b C,
$$

with $\Delta c / c_{0}=\Delta a / a_{0}=\varepsilon, C$ is the concentration of point defects and an expansion (contraction) coefficient $b$ that was derived to be ${ }^{21}$

$$
b=1 / 3\left[1-\left(r_{s} / r_{h}\right)^{3}\right] N^{-1} .
$$

Here, $N$ is the concentration of lattice sites of the host matrix $\left(8.8 \times 10^{22} \mathrm{~cm}^{-3} \mathrm{Ga}+\mathrm{N}\right.$ sites in $\left.\mathrm{GaN}\right), r_{h}$ is the covalent tetrahedral radius of host atoms and $r_{s}$ is the radius of solute atoms. Numerical values were given by Pauling ${ }^{22}$ and they neglect lattice relaxation effects and the ionicity of the constituents. The strain described by Eqs. (3) and (4) can be linearly superimposed to give the effective strain values $\varepsilon_{c, \mathrm{eff}}=\left[c-\left(c_{0}-\Delta c\right)\right] /\left(c_{0}-\Delta c\right)$ and $\varepsilon_{a, \mathrm{eff}}=\left[a-\left(a_{0}-\Delta a\right)\right] /\left(a_{0}\right.$ $-\Delta a$ ) that can be rewritten in the form

$$
\begin{gathered}
\varepsilon_{c}=(1-b C)\left(1-2 \nu \sigma_{a} / E\right)-1, \\
\varepsilon_{a}=(1-b C)\left[1+(1-\nu) \sigma_{a} / E\right]-1 .
\end{gathered}
$$

Equations (5) allow us to calculate the strain in the $\mathrm{GaN}$ films for varying biaxial stress if point defects of a given concentration are present. In Fig. 2(a) examples are given for the common substitution $\mathrm{Si}_{\mathrm{Ga}}\left(r_{\mathrm{Si}}=0.117 \mathrm{~nm}, r_{\mathrm{Ga}}=0.126\right.$ $\mathrm{nm})$ and for the two extreme substitutions $\mathrm{Ga}_{\mathrm{N}}\left(r_{\mathrm{N}}=0.07\right.$ $\mathrm{nm})$ and $\mathrm{N}_{\mathrm{Ga}}$. The elastic constants determine the slope of the lines and the incorporation of point defects induces a parallel line displacement. In addition, the largely different covalent radii of the $\mathrm{Ga}$ and the $\mathrm{N}$ atoms can easily cause changes of $\varepsilon_{c}$ in the $10^{-3}$ range if the material is nonstoichiometric. Figure 2(b) depicts that $\varepsilon_{c} / \varepsilon_{a}$ reaches values $-1.5<\varepsilon_{c} / \varepsilon_{a}<1.5$ that exceed the limits of pure hydrostatic or pure biaxial strain and compare well with Fig. 1. Thus, $\varepsilon_{c} / \varepsilon_{a}$ greatly depends on the amount of biaxial stress, the concentration of impurities and the ratio $r_{s} / r_{h}$.

This work experimentally accesses such strain related phenomena by measuring the resulting stresses by PL and by Raman spectroscopy and the strains by x-ray diffraction.

\section{EXPERIMENT}

The 5-K PL was excited at $325 \mathrm{~nm}$ by a $\mathrm{HeCd}$ laser, analyzed by a double monochromator, and detected by a lock-in amplifier. The 50-mW laser-excitation power was kept constant to provide comparable results. In this paper, the crystalline quality of the GaN films is characterized by the full width at half maximum (FWHM) of the two crystal $\mathrm{X}$-ray rocking curves. We have not attempted to extract defect concentrations from these values. Instead, we use an increasing FWHM as an indication for the degradation of the crystalline quality. $c$-lattice constants were measured with a Siemens D-5000 diffractometer equipped with a four bounce Ge monochromator and a four circle translation capability. Unpolarized, local Raman measurements were performed at room temperature by focusing a $488-\mathrm{nm}$ laser beam to a $1-\mu \mathrm{m}$ spot on the sample and detecting the back-scattered light.

Samples grown by three different methods are considered in this study.

(1) GaN films of $\sim 0.5 \mu \mathrm{m}$ thickness were grown by MBE at $675^{\circ} \mathrm{C}$. Heteroepitaxy was performed on $\mathrm{SiC}$ and on sap-
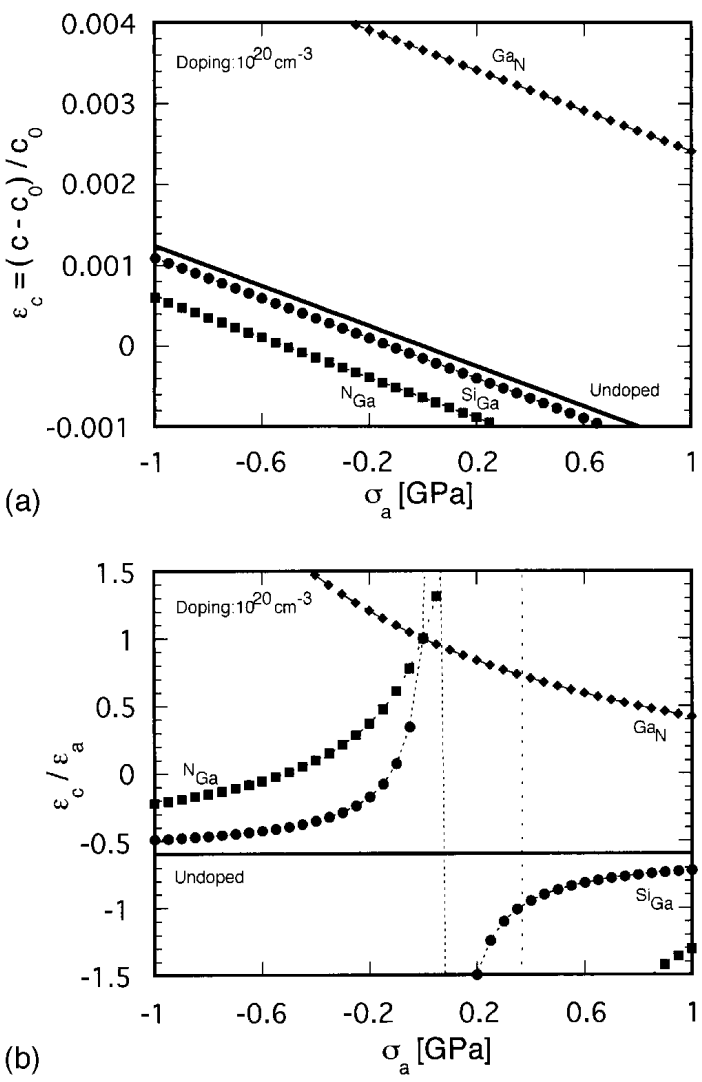

FIG. 2. (a) Calculated dependence of $\varepsilon_{c}$ on the biaxial stress $\sigma_{a}$ for selected point defects of the given concentration. Eq. (5a) was used. It can be seen that the coexisting hydrostatic and biaxial strain fields induce a parallel displacement of the lines that is positive if the size of the impurities exceeds the size of the host atom. The slope of the lines is determined solely by the elastic constants. (b) Calculated dependence of $\varepsilon_{c} / \varepsilon_{a}$ on the biaxial stress $\sigma_{a}$ for the selected point defects of the given concentration. Equations (5) were used. It can be seen that the coexisting hydrostatic and biaxial strain fields easily induce changes $-1.5<\varepsilon_{c} / \varepsilon_{a}<1.5$. A comparison with Fig. 1 directly reveals that the observed experimental scatter is explained by this effect. Numbers depend on the size of the atoms and their concentration. In case of a stoichiometric and undoped crystal one would expect a constant value that was chosen to be 0.6 because of the results presented in Fig. 1. This value is related to the Poisson ratio by Eq. (3c).

phire $c(0001)$ planes, homoepitaxy on bulk GaN. ${ }^{23,24}$ The films were undoped and $n$-type $\left(10^{16}-10^{19} \mathrm{~cm}^{-3}\right)$. Typical impurities are $\mathrm{H}, \mathrm{C}$, and $\mathrm{O}$ that can be present in concentrations as large as $10^{19} \mathrm{~cm}^{-3}$. Low-temperature (LT) GaN buffer layers were typically grown around $500{ }^{\circ} \mathrm{C}$.

(2) The two investigated $n$-type metal-organic chemicalvapor deposition (MOCVD) films were deposited at $1030{ }^{\circ} \mathrm{C}$ on AlN buffer layers. They were 3-5 $\mu \mathrm{m}$ thick and grown on sapphire $a(11-20)$-plane substrates. One of the samples was undoped and the other one doped with $\mathrm{Si}\left(3 \times 10^{18}\right.$ $\left.\mathrm{cm}^{-3}\right) .^{25}$

(3) $n$-type, $\sim 3 \mu \mathrm{m}$-thick MOCVD films were also deposited above $1000{ }^{\circ} \mathrm{C}$. In one case a LT GaN buffer layer was deposited on sapphire ${ }^{26}$ and in other experiments $\mathrm{SiC}$ $c$-plane substrates were used.

We observed cracks in none of the investigated thin films. 


\section{A. Photoluminescence and Raman studies}

Figures 3(a) and 3(b) show the near-band-edge PL spectra of $\mathrm{GaN}$ grown on $\mathrm{SiC}$ and on sapphire. A collective displacement of the PL lines up to $30 \mathrm{meV}$ can be seen. It is larger if $\mathrm{GaN}$ is grown on sapphire. Since the lines tend to broaden with increasing FWHM of the films, they overlap at some point in the case of growth on $\mathrm{SiC}$. In such a situation we determined the line position by fitting two Lorentzian lines to the data. In Fig. 3(c) we compare the PL spectrum of bulkgrown GaN with the one of a homoepitaxial film. ${ }^{24}$ The differences are remarkable. While the MBE-grown film exhibits a sharp donor bound exciton and negligible yellow luminescence, the 2.3-eV luminescence dominates greatly in bulk $\mathrm{GaN}$ and the band-edge luminescence is blueshifted and weak. TEM reveals a low dislocation density $\left(<10^{7} \mathrm{~cm}^{-2}\right)$ in the homoepitaxial film and no detectable dislocations in the bulk material near the substrate/film interface. ${ }^{27}$

The energetic position of the near-band-edge exctitons is plotted versus the FWHM of the x-ray rocking curves of GaN films grown on $\mathrm{SiC}$ with a $\mathrm{LT} \mathrm{GaN}$ buffer layer in Fig. 4(a). The linear regression to the data emphasizes that the line displacements are not statistical but a collective shift that is attributed to the presence of stress. It is noted that the yellow luminescence band shifts even stronger but those data will be evaluated elsewhere. For comparison, the position of the donor-bound exciton in a homoepitaxially grown sample is plotted too, because it provides a calibration for strain-free growth: PL energies of the donor-bound excitons smaller than $3.467 \mathrm{eV}$ indicate the presence of tensile stress, larger values indicate that the films are compressed.

In Fig. 4(b) we evaluate the PL data obtained from films grown on sapphire substrates with different buffer layers. It can be seen that the growth of a LT GaN buffer layer leads to an effective strain relaxation that is almost independent on the defect density and the growth method. In contrast, MBE growth of $\mathrm{GaN}$ films without buffer layers introduces large tensile stresses into films of poor crystalline quality. MOCVD growth on an AIN buffer layer introduces large compressive stresses. In these particular films, part of that large compressive stress could be caused by the larger lattice mismatch of the sapphire $a$ planes $(20 \%)$ compared with the growth on $c$ planes that was used for the orientation of the substrates in all other cases. However, the near-band-edge PL lines of thin GaN films $(<10 \mu \mathrm{m})$ grown on sapphire $c$ planes with AlN buffer layers also appear at energies as large as 3.48-3.49 eV as documented in an earlier study. ${ }^{28}$ Thus, the growth on an AlN buffer layer may introduce larger stresses than the growth on a LT GaN buffer layer in spite of the similar growth temperature $\left(\sim 1030{ }^{\circ} \mathrm{C}\right)$. Since in addition the MBE growth on an LT GaN buffer layer proceeds at only $675^{\circ} \mathrm{C}$, yet results in a similarly low-strained GaN film, we conclude that, beside the different thermal expansion coefficients of $\mathrm{GaN}$ and sapphire, details of the buffer-layer growth must modify the stress in the GaN films greatly.

There are several sources that can cause confusion as to a physical identification of PL transitions and the origin of their shift: different donor-bound excitons have been observed in the near-band-edge region; ${ }^{29,30}$ free-excitonic transitions are commonly observed, ${ }^{5,29,30}$ the near-band-edge PL line shifts can be caused by the Burstein-Moss effect rather
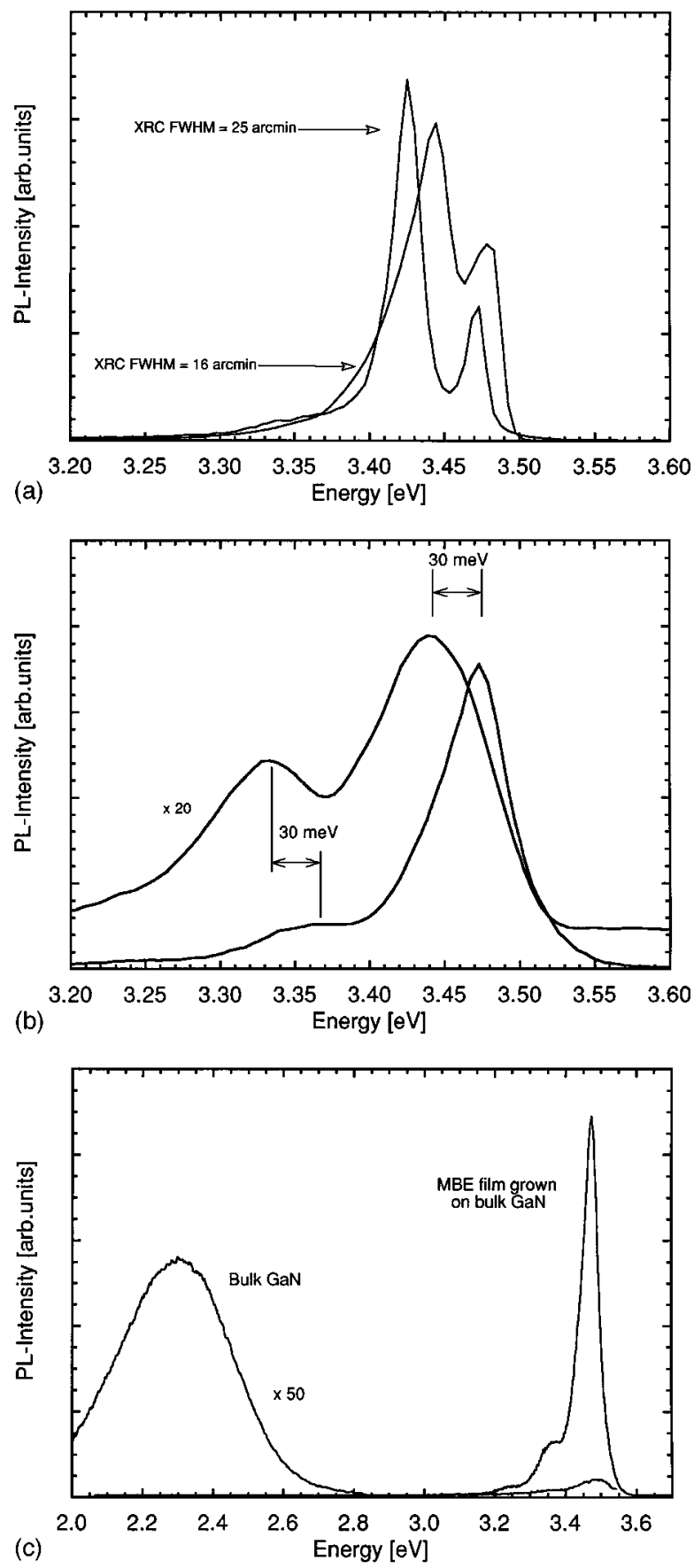

FIG. 3. (a) 5-K PL spectra of GaN films on SiC. LT GaN buffer layers were grown. A collective displacement of the PL lines by $\sim 10 \mathrm{meV}$ is seen. The $\mathrm{x}$-ray FWHM characterizes the crystalline quality of the films. There is a tendency that films grown on SiC exhibit a redshift of the PL lines. (b) 5-K PL spectra of MBE-grown GaN films on sapphire. No buffer layers were grown. A collective displacement of the PL lines by $\sim 30 \mathrm{meV}$ is seen. There is a tendency that films grown on sapphire exhibit a blueshift of the PL lines. (c) 5-K PL spectra of a homoepitaxially MBE-grown GaN film on bulk GaN. The thin film exhibits almost only donor-boundexcitonic transitions and very weak yellow luminescence that, in contrast, dominates the PL spectrum of the bulk crystal. A large blueshift of the weak near-band-edge luminescence is observed in bulk GaN. Out of all investigated crystals, only the bulk crystals exhibit a large blueshift of the near-band-edge PL that is caused by the large $n$ doping $\left(\sim 10^{20} \mathrm{~cm}^{-3}\right)$. 

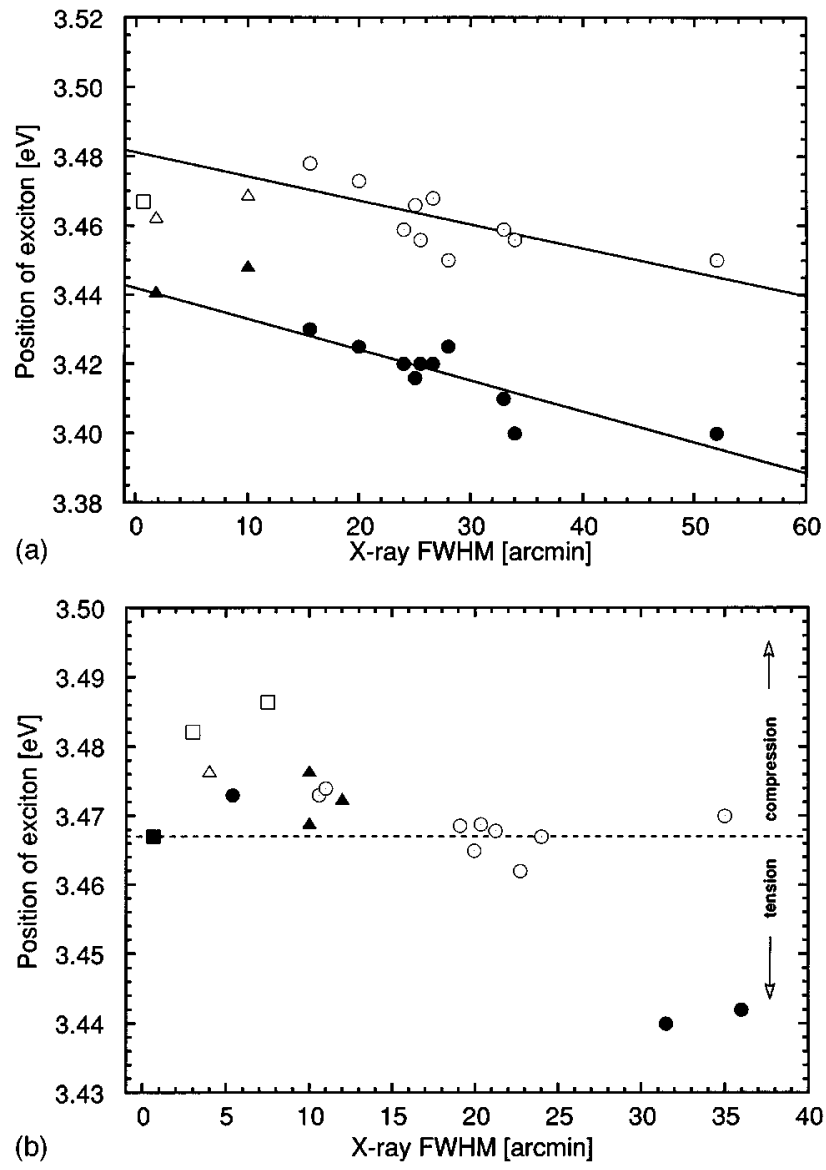

FIG. 4. (a) Energetic position of the two PL transitions from Fig. 1(a) observed in films with different crystalline quality that degrades with increasing FWHM of the x-ray rocking curves. All samples were grown on $\mathrm{SiC} c$ planes. LT GaN buffer layers were grown. The linear regression emphasizes the collective shift of the lines. Open symbols, donor-bound excitons; filled symbols, line of unknown origin; circles, MBE heteroepitaxy; square, MBE homoepitaxy; triangles, MOCVD heteroepitaxy. (b) Energetic position of the donor bound excitonic luminescence from Fig. 1(b) observed in films with different crystalline quality that degrades with increasing FWHM of the x-ray rocking curves. The films were grown on sapphire and typically on $c$ planes. It is seen that the position of the luminescence is determined by the choice of the buffer layers that greatly modifies the stress. Homoepitaxy provides a standard for stress-free growth. A redshift is caused by tensile stress and a blue shift by compression. Open circles, MBE films grown on a LT GaN buffer layer; filled circles, MBE films grown without a buffer layer; triangles, MOCVD-grown films on a LT GaN buffer layer from two different sources; filled square, homoepitaxially MBE-grown film; open squares, MOCVD films grown on sapphire $a$ planes and on an AlN buffer layer (Ref. 16). The undoped sample exhibits a larger blueshift than the doped one $\left(\mathrm{Si}: 3 \times 10^{18} \mathrm{~cm}^{-3}\right)$.

than by strain. ${ }^{31}$ Therefore, we support our assignment of the PL line shifts to the presence of stress by performing Raman measurements of the $E_{2}$ Raman mode that is known to be shifted by stress only. ${ }^{32}$ The three examples of Fig. 5 depict the presence of a tensile stress in a film grown on $\mathrm{SiC}$ and a larger compressive stress in a $\mathrm{GaN}$ film grown on sapphire. However, the $E_{2}$ line position varies almost continuously even if different samples were grown at the same tempera-

\section{GaN $E_{2}$ phonon}

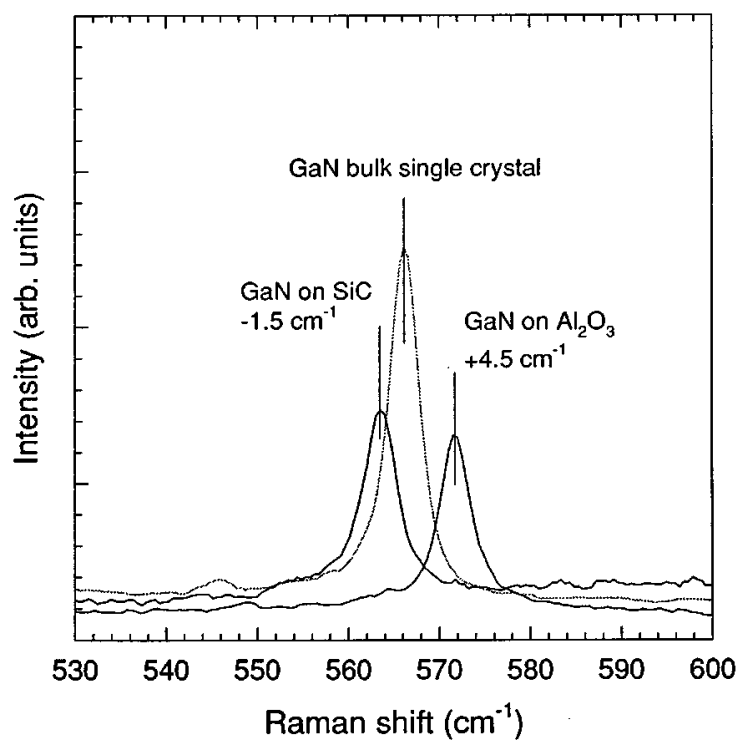

FIG. 5. Position of the $E_{2}$ Raman mode in bulk GaN single crystals and in films grown on $\mathrm{SiC}$ and on sapphire. Homoepitaxially grown MBE films and bulk GaN exhibit a position of the $E_{2}$ Raman mode at $566.2 \mathrm{~cm}^{-1}$ (stress-free standard). The shifts of the line position are caused by a tensile stress in the film grown on $\mathrm{SiC}$ and compressive one if grown on sapphire.

ture. Again, the stress cannot exclusively be determined by the thermal expansion coefficients of the films and the substrates. Moreover, the $E_{2}$ Raman mode of homoepitaxially grown $\mathrm{GaN}$ and the investigated bulk grown GaN appears at an identical wave number of $566.2 \mathrm{~cm}^{-1}$. We take this number as a reference for unstressed films. However, it is noted that the lattice constant of bulk GaN may locally vary, ${ }^{18}$ which is why this identity can be accidental.

Next, a calibration of the Raman and the PL line shifts is established for stress-free, biaxially, and hydrostatically stressed samples. Again, we assume that the homoepitaxially MBE-grown GaN film deposited on bulk GaN is not biaxially strained because of the identical thermal expansion coefficients of the film and the substrate. The donor bound exciton energy $E_{0}=3.467 \mathrm{eV}$ and the $E_{2}$ Raman mode at $\omega_{0}=566.2 \mathrm{~cm}^{-1}$ determine the calibration constant $k=E_{0} / \omega_{0}=6.12 \times 10^{-3} \mathrm{eV} \mathrm{cm}$. For biaxially stressed films, a relation $E\left(\sigma_{a}\right)=k_{a} \omega\left(\sigma_{a}\right)$ is expected where $\sigma_{a}$ is a biaxial stress. Therefore, we plot in Fig. 6 the energy position of the donor bound excitons versus the measured position of the $E_{2}$ Raman mode for our samples that exhibit different amounts of shifts. A linear regression to the data yields the calibration constant $k_{a}=6.6 \times 10^{-3} \mathrm{eV} \mathrm{cm}$. The scatter of the data in Fig. 5 was found to be caused by lateral inhomogeneities in the GaN films. Finally, a hydrostatic pressure $\sigma_{p}$ has been measured to shift the $E_{2}$ Raman mode by $\omega=\omega_{0}+3.6$ $\mathrm{cm}^{-1} \mathrm{GPa}^{-1} \sigma_{p}$ and the near-band-edge PL lines by $E=E_{0}+40 \mathrm{meV} \mathrm{GPa}^{-1} \sigma_{p} \cdot{ }^{33,34}$ The ratio $k_{p}=40 \mathrm{meV}$ $\mathrm{GPa}^{-1} / 3.6 \mathrm{~cm}^{-1} \mathrm{GPa}^{-1}=1.110^{-2} \mathrm{eV} \mathrm{cm}$ is the calibration constant for the hydrostatic case. The relation $E\left(\sigma_{p}\right)=k_{p} \omega\left(\sigma_{p}\right)$ is depicted in Fig. 6 too. $k_{p}$ is almost twice as large as the $k_{a}$ measured in our experiments. We assign 


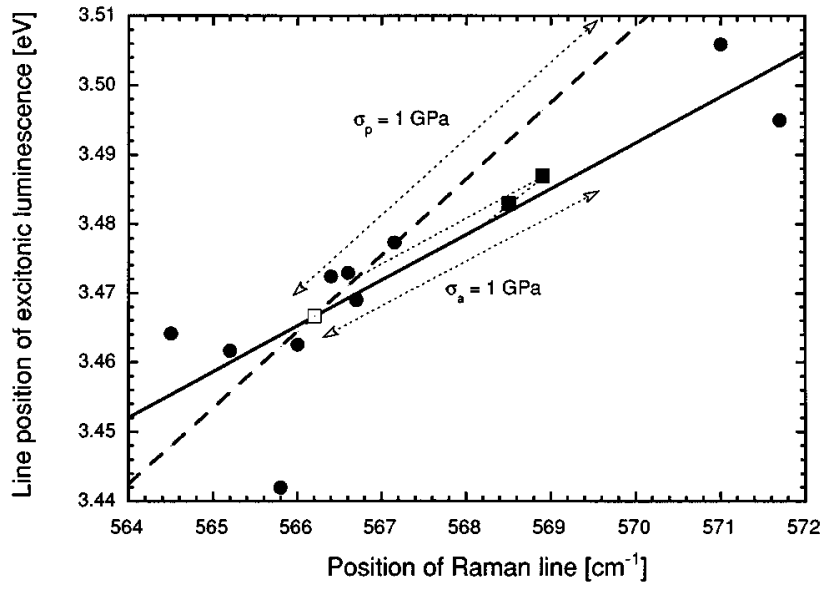

FIG. 6. Plot of the donor-bound PL energy versus the position of the $E_{2}$ Raman mode. A linear regression to the data yields the calibration constant $k_{a}=6.610^{-3} \mathrm{eV} \mathrm{cm}$ (solid line). The dashed line is calculated from the known hydrostatic pressure dependence of the PL and the Raman lines $\left(k_{p}=1.1 \times 10^{-2} \mathrm{eV} \mathrm{cm}\right)$. The homoepitaxial film (open square) provides the calibration for the stress-free case. The absolute stress calibration is given for one GPa. In the absence of experimental errors, a difference between the biaxial and the hydrostatic stress can be determined by the indicated projections on the straight lines as indicated for the two MOCVD samples (squares).

this discrepancy to the biaxial nature of the stresses $\sigma_{a}$ in the GaN films. It can be seen from Fig. 6 that most of the experimental data are influenced by both a hydrostatic and a biaxial strain component as one would expect from the review of the data presented in Fig. 1.

Principally, the results presented in Fig. 6 allow for an extraction of differences between hydrostatic and biaxial stresses by projecting the data points on each of the straight lines. Figure 6 gives an example. It refers to the Si-doped and undoped MOCVD-grown samples. It can be seen that both samples hold a similar amount of biaxial stress $\sigma_{a}=0.6$ $\mathrm{GPa}$. In contrast, the Si-doped sample exhibits a reduction of $\sigma_{p}$ by less than $0.1 \mathrm{GPa}$.

Obviously, the procedure requires an absolute calibration of the hydrostatic and biaxial stresses. This calibration is readily given for the hydrostatic pressure because $\sigma_{p}$ has been measured to shift the PL lines by $40 \mathrm{meV} \mathrm{GPa}^{-1}$ and the $E_{2}$ Raman mode by $3.6 \mathrm{~cm}^{-1} \mathrm{GPa}^{-1}$. The biaxial stress is calibrated in the next section but the result is already indicated in Fig. 6.

\section{B. Absolute calibration of the biaxial stress}

The PL and the Raman line shifts are compared with $c$-lattice constant measurements in Figs. 7(a) and 7(b). A large scatter of the data can be seen. We expect that Eq. (3b) is valid. A linear relation between the biaxial stress $\sigma_{a}$ and the Raman and PL line shifts is assumed: $\sigma_{a}=-\Delta \omega / k_{\mathrm{Ra}, a}$ and $\sigma_{a}=-\Delta E / k_{\mathrm{PL}, a}$. Here the negative sign accounts for a positive line shift that is induced by a negative (compressive) stress. $k_{\mathrm{Ra}, a}$ and $k_{\mathrm{PL}, a}$ are the absolute calibration constants for the Raman shift $\Delta \omega$ and the PL line shift $\Delta E$ induced by a biaxial stress. Thus, we expect the relations $\varepsilon_{c}$ $=E^{-1} 2 \nu \Delta \omega / k_{\mathrm{Ra}, a}$ and $\varepsilon_{c}=E^{-1} 2 \nu \Delta E / k_{\mathrm{PL}, a}$ to be valid for
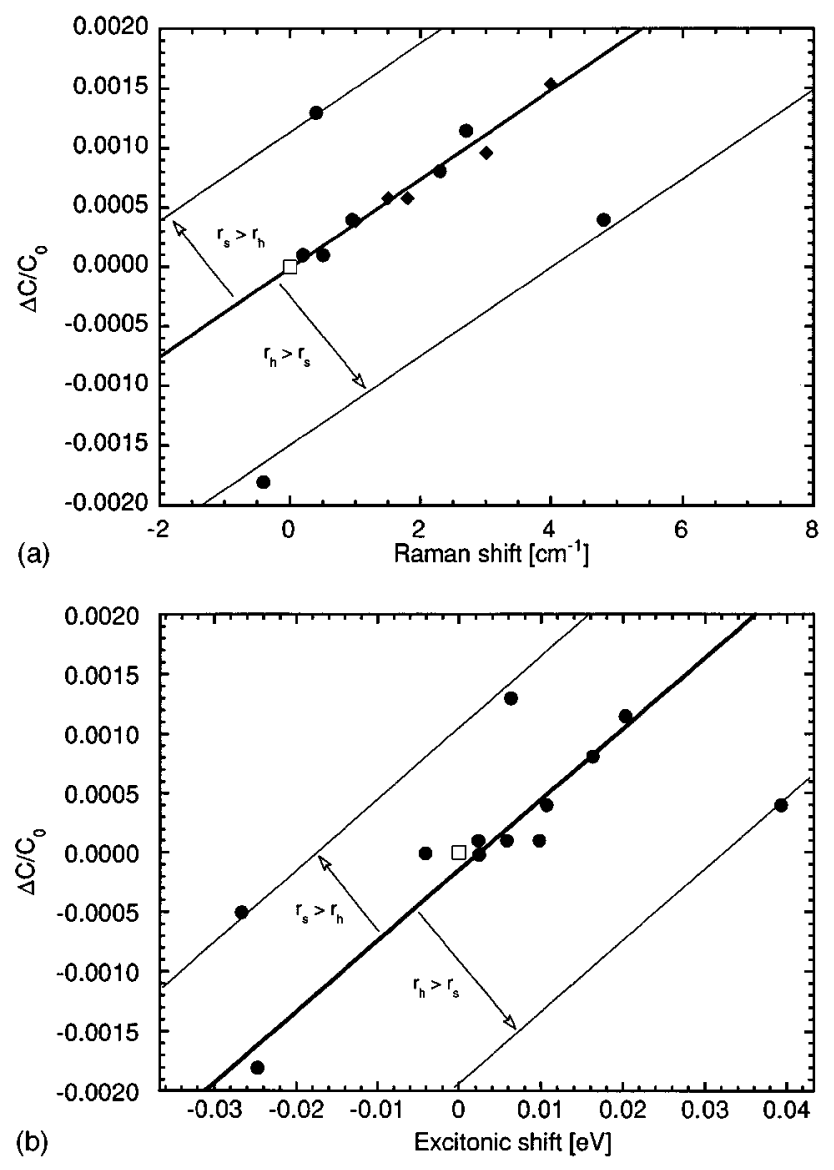

FIG. 7. (a) Plot of $\left(c-c_{0}\right) / c_{0}$ versus the Raman shift of the $E_{2}$ mode. A positive Raman shift indicates compressive (negative) stress. The bold straight line is fitted to achieve an absolute calibration of the biaxial stress. Data close to the thin lines were excluded from fitting. The parallel displacement of such data points is attributed to large hydrostatic strain fields caused by point defects. A positive line displacement is caused by the larger size of solute atoms compared with host atoms $\left(r_{s}>r_{h}\right)$. Filled circles, this work. Filled squares, data extracted from Ref. 19. Open square, homoepitaxial MBE growth. (b) Plot of $\left(c-c_{0}\right) / c_{0}$ versus the shift of the excitonic luminescence. The plot compares with (a).

films with a dominant contribution of a biaxial stress to the line shifts. A linear regression to those data in Fig. 7 that do not significantly deviate from a straight line yields $\varepsilon_{c}=3.8 \pm 0.4 \times 10^{-4} \mathrm{~cm} \Delta \omega$ and $\varepsilon_{c}=(0.059 \pm 0.006) \mathrm{eV}^{-1} \Delta \mathrm{E}$ for the Raman and the PL measurements, respectively. It can be seen from Fig. 7 that both linear regressions closely match the zero calibration point given by the homoepitaxially grown GaN films. A comparison with Fig. 2(a) reveals that in this case all of the evaluated films are mainly biaxially strained. On the other hand, the data which were excluded from the regression can be described by parallel straight lines and their offset is attributed to an incorporation of point defects with tetrahedral covalent radii that are either smaller or larger than the radii of the host atoms [compare with Eq. (5a) and with Fig. 2(b)].

The slopes of the lines and the isotropic elastic constants from Table I determine $k_{\mathrm{Ra}, a}^{-1}=4.2 \pm 0.3 \mathrm{~cm}^{-1} \mathrm{GPa}^{-1}$ and $k_{\mathrm{PL}, a}^{-1}=27 \pm 2 \mathrm{meV} \mathrm{GPa}^{-1}$. The uncertainties in $B$ and $\nu$ are neglected in the given error bars. The quotient $k_{\mathrm{PL}, a} / k_{\mathrm{Ra}, a}$ 
$=6.6 \times 10^{-3} \mathrm{eV} \mathrm{cm}$ equals $k_{a}=6.6 \times 10^{-3} \mathrm{eV} \mathrm{cm}$ that was determined from Fig. 5. Thus, we obtained consistency between the quotient of the absolute calibration constants $k_{\mathrm{PL}, a} / k_{\mathrm{Ra}, a}$ obtained from x-ray measurements with the relative calibration constant $k_{a}$ deduced from the direct comparison of the PL and Raman measurements. In particular, the values $k_{\mathrm{PL}, a}^{-1}$ and $k_{\mathrm{Ra}, a}^{-1}$ scale the biaxial strain in Fig. 6 absolutely.

\section{PL intensity measurements}

Further evidence for a varying concentration of point defects in the investigated samples comes from intensity measurements. The intensity ratio of the yellow luminescence band at $2.3 \mathrm{eV}$ and the near-band-edge luminescence of the $\mathrm{GaN}$ films is analyzed. These luminescence lines are of special interest because of their possible relation to the presence of native point defects. ${ }^{10,34}$ The lines are observed in bulk-, MBE-, and MOCVD-grown samples. This supports the intrinsic character of the defects that give rise to the PL lines. Figure 8 reveals that their ratio varies the most in the case of high-quality GaN films (FWHM $<10$ arc min): in homoepitaxially MBE-grown GaN the yellow luminescence can drop down to the detection limit or appear at medium ratios depending on the growth conditions; in the MOCVD crystals, medium ratios were detected too; in bulk $\mathrm{GaN}$ the ratio is the largest. Thus, the defect introduction depends on growth parameters. In crystals with poorer quality the ratio scatters considerably also. On the average, however, it is smaller if the GaN films were grown on SiC. This was reported earlier for a growth series that compared $\mathrm{GaN}$ growth on $\mathrm{SiC}$ with growth on sapphire. ${ }^{35}$ The average ratio drops with degrading crystalline quality. Therefore, the PL spectra of crystals with very poor quality may also exhibit only near-band-edge luminescence and no yellow luminescence band.

\section{DISCUSSION AND CONCLUSION}

Our experiments were triggered by the observed large strain induced shifts of the near-band-edge PL that causes confusion as to an identification of the involved transitions. However, attempts to understand the phenomena soon revealed serious limitations such as the inconsistency of the anisotropic elastic constants (Table I) or the unexpected variation of the $\varepsilon_{c} / \varepsilon_{a}$ values (Fig. 1) that does not change even if the films crack. ${ }^{16}$ In particular, it is the range of the measured $\varepsilon_{c} / \varepsilon_{a}$ ratios that made us doubt the common belief that it is only the biaxial strain that is responsible for the spectroscopic line shifts.

The presented model rationalizes the large scatter of $\varepsilon_{c} / \varepsilon_{a}$. We stress four facts. First, the anisotropic elastic constants can be compared with the experimentally deduced isotropic elastic constants by calculating the Poisson ratio, the Young $(E)$ and the bulk $(B)$ modulii in the Voigt average from the anisotropic elastic constants ${ }^{36}(z$ along the $c$ axis and $x, y$ in the basal plane). They are listed in Table I. It can be seen that the data from Refs. 13 and 15 compare well with our values. Moreover, the ratio $\varepsilon_{c} / \varepsilon_{a}=-2 c_{13} / c_{33}=-0.54$ from Ref. 15 agrees closely with our isotropic estimation $\varepsilon_{c} / \varepsilon_{a}=-2 \nu /(1-\nu)=-0.6+0.2$ extracted from Fig. 1 . Thus, we find the best agreement of our isotropic elastic
TABLE II. A dependence of the Raman calibration factor $k_{\mathrm{Ra}, a}$ on the isotropic elastic constants is given by Eq. (3b). Here, we evaluate the effect of different isotropic elastic constants on the Raman calibration factor. Typical values from literature are taken. For details see text.

\begin{tabular}{lccc}
\hline \hline $\begin{array}{l}\text { Poisson } \\
\text { ratio }\end{array}$ & $\begin{array}{c}\text { Bulk } \\
\text { modulus } \\
(\mathrm{GPa})\end{array}$ & $\begin{array}{c}\text { Young } \\
\text { modulus } \\
(\mathrm{GPa})\end{array}$ & $\begin{array}{c}\text { Raman } \\
\text { factor } \\
{\left[(\mathrm{GPa} \mathrm{cm})^{-1}\right]}\end{array}$ \\
\hline 0.23 & 200 & 290 & 4.2 \\
0.37 & 200 & 180 & 10.8 \\
0.30 & 163 & 196 & 8.1 \\
\hline \hline
\end{tabular}

constants with the anisotropic elastic constants from Ref. 15. Second, the factor $[-2 \nu /(1-\nu)]$ determines $\left[\left(c-c_{0}\right) / c_{0}\right] /$ $\left[\left(a-a_{0}\right) / a_{0}\right]$ in the absence of point defects [Fig. 2(b)]. It is the presence of nonstoichiometric defects or impurities that makes $\varepsilon_{c} / \varepsilon_{a}$ vary greatly. From Figs. 2(a) and 7 one could expect that their concentration can exceed $10^{19} \mathrm{~cm}^{-3}$. This limits the accuracy to which $\nu$ can be determined by measuring the lattice constants $c$ and $a$ of GaN thin films if the stoichiometry is uncertain. Third, it is the unusual large difference of the covalent tetrahedral radii of $\mathrm{Ga}$ and $\mathrm{N}$ that can cause large changes of the lattice constants in case of a moderate introduction of nonstoichiometric defects or impurities. Finally, homoepitaxial growth of $\mathrm{GaN}$ recently provided benchmark values for crucial parameters such as the zero stress value for the donor-bound exciton ${ }^{5,24}$ and the $E_{2}$ Raman mode or for the reference lattice constants $a_{0}$ and $c_{0} .{ }^{16}$

The presented data analysis assumes a statistical fluctuation of the hydrostatic strain components that are induced by point defects in those samples that exhibit a dominant biaxial stress. We calculate the mean value $\varepsilon_{c} / \varepsilon_{a}=-0.6+0.2$ and the slopes in Fig. $6, \varepsilon_{c}=3.8 \pm 0.410^{-4} \mathrm{~cm} \Delta \omega$ and $\varepsilon_{c}=0.059 \pm 0.006 \Delta E$ with this assumption. Its validity is supported by the result of the linear regressions in Fig. 6 that closely match the zero calibration point provided by homoepitaxial growth. This would be difficult if the point defects in the investigated samples would induce a hydrostatic strain that is not zero on the average.

At present, the proposed model allows for a qualitative comparison with experimental data only, because of various reasons. First, any uncertainty in the isotropic elastic constants strongly modifies the given calibration constants. This is demonstrated for the Raman calibration constant $k_{\mathrm{Ra}, a}$ in Table II. The first line lists the values we consider best. In the second line we calculated $k_{\mathrm{Ra}, a}$ with a Poisson ratio $\nu=0.37$ that is used in the literature (Table I). It can be seen that this $60 \%$ change of $\nu$ causes $k_{\mathrm{Ra}, a}$ to change by $160 \%$. In the third line we use the elastic constants from Ref. 37. $k_{\mathrm{Ra}, a}=6.2 \mathrm{~cm}^{-1}$ was reported there. It can be seen that we would calculate a comparably large value of $8.1 \mathrm{~cm}^{-1}$ from these elastic constants. Therefore, we conclude that it is the poor knowledge of the elastic constants that induces the largest uncertainties. The experimental uncertainty in $\nu=0.23$ \pm 0.06 provided in this paper still exceeds the uncertainties obtained from the measurements of Fig. 6 that give $k_{\mathrm{Ra}, a}$ $=4.2 \pm 0.3 \mathrm{~cm}^{-1} \mathrm{GPa}^{-1}$ and $k_{\mathrm{PL}, a}^{-1}=27 \pm 2 \mathrm{meV} \mathrm{GPa}^{-1}$ if we neglect the uncertainties in $\nu$ and $B$. Second, the calculation 
of the lattice expansion/contraction induced by point defects neglects lattice relaxation effects and the ionicity of the constituents. Thus, it will systematically overestimate the size effect.

In Fig. 6 we show that a hydrostatic and a biaxial stress can uniquely be determined from the PL and the Raman measurements. Physically, we distinguish between them because the biaxial stress is induced through the substrate but the additional hydrostatic strain variations by the presence of point defects. Principally, however, the biaxial stress can be decomposed into a hydrostatic stress and a uniaxial stress, which is why the information about hydrostatic components of different origins is mixed in Fig. 6. Again modeling is required to extract useful information. This problem will be treated elsewhere because, at present, the poor quality of the GaN films causes broadening of the PL transitions and thereby line superpositions that give rise to hardly controllable uncertainties which we expect to decrease rapidly with improving crystal quality of the GaN films.

Our analysis does not explicitly address strain relaxation processes that are induced by buffer layers or by dislocation networks. The lattice mismatch, the thermal strain and the effect of buffer layers and of dislocation networks on the lattice relaxation are simply expected to modify measured biaxial stresses. However, it is of practical importance for crystal growth to realize that the growth of a LT GaN buffer layer relaxed the stresses most efficently in our investigated samples [Fig. 3(b)] for reasons that are currently under investigation.

We focused our study on investigating the influence of point defects on the lattice parameters. Evidence for the modification of the biaxial strain comes from the largely varying values of $\varepsilon_{c}$ and $\varepsilon_{c} / \varepsilon_{a}$ in Figs. 1 and 7 . Further evidence is presented by measuring the intensity of the nearband-edge PL and the yellow luminescence (Fig. 8). The presence of these PL lines in extended defect-free areas of bulk-grown GaN (Refs. 10 and 27) links them with point defects or defect clusters that can be inhomogeneously distributed if extended defects are present. ${ }^{27,38}$ Since they appear in all investigated samples, they seem to be of intrinsic origin or related to a common impurity. Usually, it is the strain field around dislocations with edge character that interacts with point defects to form a Cotrell atmosphere. ${ }^{36}$ Thus, the inhomogeneous distribution of the luminescence intensity can be the result of either of the two processes that minimize the strain energy: either point defects are gettered around dislocations or they are preferentially formed during growth to locally or globally relaxed strain. Finally, it has recently been shown ${ }^{39}$ that the strain in the layers can either be changed by varying the III/V flux ratio during growth that induces a compositional change or by modifying the biaxial strain due to the growth of different buffer layers.

In conclusion, we show that in GaN thin films the superposition of a hydrostatic and a biaxial strain field determines the observed stress. A hydrostatic strain is induced by the presence of point defects and the biaxial strain by the growth on lattice mismatched substrates with different thermal ex-

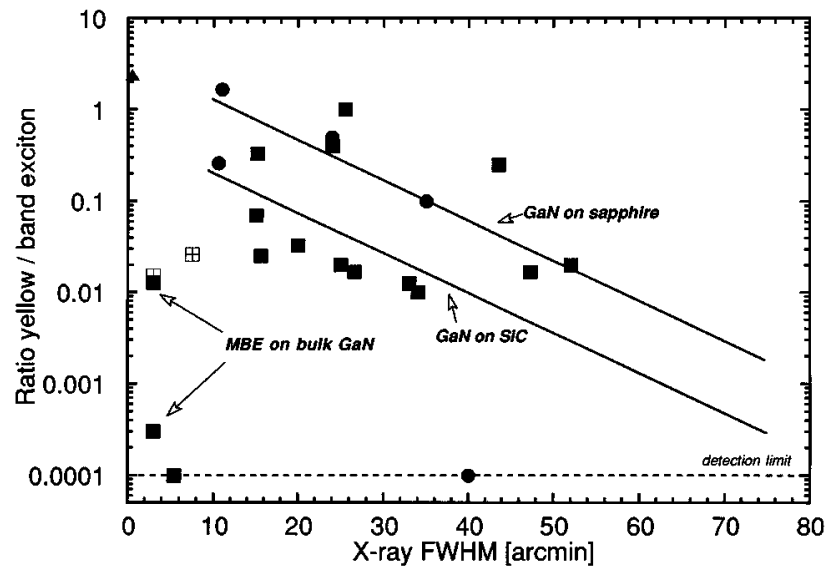

FIG. 8. Intensity ratio of the yellow luminescence band and the donor bound near-band-edge PL lines in films with different crystalline quality that degrades with increasing FWHM of the x-ray rocking curves. Filled squares, MBE-grown films on $\mathrm{SiC}$ and bulk GaN. Filled circles, MBE-grown films on sapphire. Filled triangle, bulk GaN. Crossed squares, MOCVD films grown on sapphire $a$ planes.

pansion coefficients. The presence of both strain fields limits the accuracy as to which elastic constants can be determined. A consistent description of PL, Raman, and x-ray-diffraction measurement is presented that uses a best estimation of the bulk modulus $B=200 \pm 20 \mathrm{GPa}$ and of the Poisson ratio $\nu=0.23 \pm 0.06$. Only one set of published anisotropic elastic constants ${ }^{15}$ agrees best with these isotropic elastic constants. Homoepitaxial growth of $\mathrm{GaN}$ provides calibration constants for stress-free samples. A procedure to determine differences between the biaxial and the hydrostatic stress from the PL and Raman measurements is presented. In particular, we find that a biaxial stress of one GPa would shift the $E_{2}$ Raman mode by $4.2 \pm 0.3 \mathrm{~cm}^{-1} \mathrm{GPa}^{-1}$ and the near-band-edge PL lines by $27 \pm 2 \mathrm{meV} \mathrm{GPa}^{-1}$. The dependence of these values on the isotropic elastic constants is given. Experimental evidence is presented that indicates a varying concentration of (native) point defects in the $\mathrm{GaN}$ films grown by different methods. A dependence of the biaxial stress on the buffer layer growth conditions and its modification by an intentional incorporation of (native) point defects seem to allow for strain engineering of $\mathrm{GaN}$ films.

\section{ACKNOWLEDGMENTS}

This work was supported by the Office of Energy Research, Office of Basic Energy Sciences, Division of Advanced Energy Projects (BES-AEP), and by the Laboratory Technology Transfer Program (ER-LTT) of the U.S. Department of Energy under Contract No. DE-AC03-76SF00098. S.R. and Z.L.-W. were supported by the Director, Office of Energy Research, Office of Basic Energy Science of the U.S. Department of Energy under Contract No. DE-AC0376SF00098, and T.S. by Fulbright Foundation. C.K. acknowledges fruitful discussions with P. Specht. 
*Electronic address: cfk@ux5.1b1.gov

${ }^{1}$ S. Nakamura, M. Senoh, N. Iwasa, and S. Nagahama, Jpn. J. Appl. Phys. Lett. 34, L797 (1995).

${ }^{2}$ M. A. Kahn, A. Bhattarai, J. N. Kuznia, and D. T. Olsen, Appl. Phys. Lett. 63, 1214 (1993).

${ }^{3}$ S. Nakamura, M. Senoh, S. Nagahama, N. Iwasa, T. Yamada, T. Matsushita, H. Kiyoku, and Y. Sugimoto, Jpn. J. Appl. Phys. 35, L217 (1996).

${ }^{4}$ H. Morkoc, S. Strite, G. B. Gao, M. E. Lin, B. Sverdlov, and M. Burns, J. Appl. Phys. 76, 1363 (1994).

${ }^{5}$ K. Pakula, A. Wysmolek, K. P. Korona, J. M. Baranowski, R. Stepniewski, I. Grzegory, M. Bockowski, J. Jun, S. Krukowski, M. Wroblewski, and S. Porowski (unpublished).

${ }^{6}$ F. A. Ponce, D. P. Bour, W. Götz, N. M. Johnson, H. I. Helava, I. Grzegory, J. Jun, and S. Porowski, Appl. Phys. Lett. 68, 917 (1996).

${ }^{7}$ J. Neugebauer and C. G. Van de Walle, Phys. Rev. B 50, 8067 (1994).

${ }^{8}$ P. Boguslawski, E. Briggs, and J. Bernholz, Phys. Rev. B 51, 17255 (1995).

${ }^{9}$ O. Lagerstedt and B. Monemar, Phys. Rev. B 19, 3064 (1979).

${ }^{10}$ Z. Liliental-Weber, C. Kisielowski, X. Liu, L. Schloss, J. Washburn, E. R. Weber, I. Grzegory, M. Bockowski, J. Jun, T. Suski, and S. Porowski, Solid State Electron. (to be published).

${ }^{11}$ K. Hiramatsu, T. Detchprohm, and I. Akasaki, Jpn J. Appl. Phys. 32, 1528 (1993)

${ }^{12}$ V. A. Savastenco and A. U. Sheleg, Phys. Status Solidi A 48, K135 (1978).

${ }^{13}$ K. Kim, W. R. L. Lambrecht, and B. Segall, Phys. Rev. B 50, 1502 (1994).

${ }^{14}$ T. Azuhata, T. Sota, and K. Suzuki, J. Phys. Condens. Matter 8, 3111 (1996).

${ }^{15}$ A. Polian, M. Grimsdich, and I. Grzegory, J. Appl. Phys. 79, 3343 (1996).

${ }^{16}$ T. Detchprohm, K. Hiramatsu, K. Itoh, and I. Akasaki, Jpn. J. Appl. Phys. 31, L1454 (1992).

${ }^{17}$ M. Leszczynski, T. Suski, P. Perlin, H. Teisseyre, I. Grzegory, M. Bockowski, J. Jun, S. Porowski, and J. Major, J. Phys. D 20, A149 (1995).

${ }^{18}$ M. Leszczynski, H. Teisseyre, T. Suski, I. Grzegory, M. Bockowski, J. Jun, S. Porowski, K. Pakula, and J. M. Baranowski (private communication).

${ }^{19}$ W. Riger, T. Metzger, H. Angerer, R. Dimitrov, O. Ambacher, and M. Stutzmann, Appl. Phys. Lett. 68, 970 (1996).

${ }^{20}$ See, e.g., A. Kelly and G. W. Groves, Crystallography and Crystal Defects (Addison-Wesley, Reading, MA, 1970).

${ }^{21}$ Y. T. Lee, N. Miyamoto, and J.-I. Nishizawa, J. Electrochem. Soc. 122, 530 (1975).

${ }^{22}$ L. Pauling, The Chemical Bond (Cornell University Press, Ithaca, 1967).
${ }^{23}$ N. Newman, J. Ross, and M. Rubin, Appl. Phys. Lett. 62, 1242 (1993).

${ }^{24}$ A. Gassmann, T. Suski, Z. Liliental-Weber, N. Newman, C. Kisielowski, E. Jones, E. R. Weber, M. D. Rubin, H. I. Helava, I. Grzegory, M. Bockowski, J. Jun, and S. Porowski, J. Appl. Phys. 80, 1 (1996).

${ }^{25}$ S. Ruvimov, Z. Liliental-Weber, T. Suski, J. W. Ager III, J. Washburn, J. Krüger, C. Kisielowski, E. R. Weber, H. Amano, and I. Akasaki, Appl. Phys. Lett. 69, 990 (1996).

${ }^{26}$ S. Nakamura, M. Senoh, N. Iwasa, S. Nagahama, T. Yamada, and T. Mukai, Jpn. J. Appl. Phys. 34, L1332 (1995).

${ }^{27}$ Z. Liliental-Weber, S. Ruvimov, C. Kisielowski, Y. Chen, W. Swider, J. Washburn, N. Newman, A. Gassmann, X. Liu, L. Schloss, E. R. Weber, I. Grzegory, M. Bockowski, J. Jun, T. Suski, K. Pakula, J. Baranowski, S. Porowski, H. Amano, and I. Akasaki, in Gallium Nitride and Related Materials, edited by R. D. Dupuis, J. A. Edmond, F. A. Ponce, and S. Nakamura, MRS Symposia Proceedings No. 395 (Materials Research Society, Pittsburgh, 1996).

${ }^{28}$ H. Amano, K. Hiramatsu, and I. Akasaki, Jpn. J. Appl. Phys. 27, L1384 (1988).

${ }^{29}$ D. Volm, K. Oettinger, T. Streibel, D. Kovalev, M. Ben-Chorin, J. Diener, B. K. Meyer, J. Majewski, L. Eckey, A. Hoffmann, H. Amano, I. Akasaki, K. Hiramatsu, and T. Detchprohm (unpublished).

${ }^{30}$ C. Merz, M. Kunzer, U. Kaufmann, I. Akasaki, and H. Amano, Semicond. Sci. Technol. 11, 712 (1996).

${ }^{31}$ H. Teisseyere, P. Perlin, M. Leszcynski, T. Suski, I. Grzegory, J. Jun, S. Porowski, and T. D. Mustakas, J. Appl. Phys. 76, 2429 (1994).

${ }^{32}$ P. Perlin, C. Jauberthie-Carillon, J. P. Itie, A. S. Miguel, I. Grzegory, and A. Polian, Phys. Rev. B 45, 83 (1992).

${ }^{33}$ P. Perlin, T. Suski, H. Teisseyre, M. Leszczynski, I. Grzegory, J. Jun, S. Porowski, P. Boguslawski, J. Bernholc, J. C. Chervin, A. Polian, and T. D. Moustakas, Phys. Rev. Lett. 75, 296 (1995).

${ }^{34}$ P. Perlin, I. Gorczyca, N. E. Christiansen, I. Grzegory, H. Teisseyre, and T. Suski, Phys. Rev. B 45, 13307 (1992).

${ }^{35}$ T. C. Fu, N. Newman, E. Jones, J. S. Chan, X. Liu, M. D. Rubin, N. W. Cheung, and E. R. Weber, J. Electron. Mater. 24, 249 (1995).

${ }^{36}$ See, e.g., J. P. Hirth and J. Lothe, Theory of Dislocations (McGraw-Hill, New York, 1960).

${ }^{37}$ T. Kozawa, T. Kachi, H. Kano, H. Nagase, M. Koide, and K. Manabe, J. Appl. Phys. 77, 4389 (1995).

${ }^{38}$ F. A. Ponce, D. P. Bour, W. Gotz, and P. J. Wright, Appl. Phys. Lett. 68, 57 (1996).

${ }^{39}$ C. Kisielowski, J. Krüger, M. Leung, R. Klockenbrink, H. Fujii, T. Suski, Sudhir G. S., J. W. Ager III, M. Rubin, and E. R. Weber, in Proceedings of the International Conference on Physics of Semiconductors, Berlin, 1996 (World Scientific, Singapore, in press). 